\title{
Prevalencia y comorbilidades de glaucoma en una institución especializada durante el año 2018
}

\section{Prevalence and comorbidities associated with glaucoma in a specialized institution during 2018}

\author{
Óscar Ariza-Pardo ${ }^{1}$, Ma. José Duque-Cardona ${ }^{1}$, Jorge L. Millán-Taborda ${ }^{1}$, Javier A. Bernal-Urrego ${ }^{2}$ y \\ Olga A. Nieto-Cárdenas ${ }^{1 *}$ \\ ${ }^{1}$ Facultad de Ciencias de la Salud, Programa de Medicina, Grupo de investigación en Salud Pública, Universidad del Quindío; ${ }^{2}$ Clínica Oftalmológica \\ Quindío. Armenia, Colombia
}

\section{Resumen}

Introducción: El glaucoma constituye un problema de salud pública que genera discapacidad visual evitable, sin embargo, se desconoce su prevalencia y factores de riesgo en el Departamento del Quindío. Objetivo: Describir características y comorbilidades del glaucoma en pacientes que consultaron a oftalmología en el Quindío durante el año 2018. Diseño del estudio: Estudio descriptivo de corte transversal. Métodos: Se tomaron las historias de los pacientes que consultaron a una institución especializada del Quindío durante el 2018. Se describieron las variables en promedio, desviación estándar e intervalos de confianza y se hizo comparación por sexo. Resultados: La prevalencia de glaucoma fue del $12.68 \%$ y el $57.71 \%$ tenía antecedente de hipertensión arterial. Conclusiones: El número de pacientes que consultan por enfermedades del ojo ha venido en aumento durante los últimos tres años en un centro especializado de la ciudad de Armenia.

Palabras clave: Glaucoma. Oftalmología. Salud pública.

\begin{abstract}
Background: Glaucoma constitutes a public health problem that generates avoidable visual impairment, however, its prevalence and risk factors in the Department of Quindío are unknown. Objective: To describe characteristics and comorbidities of glaucoma in patients who consulted ophthalmology in Quindío during 2018. Study design: Descriptive cross-sectional study. Methods: The histories of the patients who consulted a specialized institution in Quindío during 2018 were taken. The variables were described in mean, standard deviation and confidence intervals and a comparison was made by sex. Results: The prevalence of glaucoma was $12.68 \%$ and $57.71 \%$ had a history of arterial hypertension. Conclusions: The number of patients consulting for eye diseases has been increasing over the last 3 years in a specialized center in the city of Armenia.
\end{abstract}

Key words: Glaucoma. Ophthalmology. Public health.

Correspondencia:

*Olga A. Nieto-Cárdenas

E-mail: olgalicianieto@gmail.com

0120-0453 / @ 2021 Sociedad Colombiana de Oftalmología (SOCOFTAL). Publicado por Permanyer. Este es un artículo open access bajo la licencia
Fecha de recepción: 18-11-2020

Fecha de aceptación: 30-05-2021

DOI: $10.24875 /$ RSCO.M21000014
Disponible en internet: 28-12-2021 Rev Soc Colomb Oftalmol. 2021;54(2):71-75

www. revistaSCO.com CC BY-NC-ND (http://creativecommons.org/licenses/by-nc-nd/4.0/). 


\section{Introducción}

El glaucoma constituye un problema de salud pública porque genera discapacidad visual evitable, sin embargo, se desconoce la prevalencia actual, las comorbilidades en la región y su situación en el marco de las Rutas integrales de atención en Salud (RIAS) para la hipertensión y la diabetes que tienen establecida la remisión a oftalmología como seguimiento de daño a órgano blanco ${ }^{1-3}$.

El glaucoma se refiere a una enfermedad en la que el daño al nervio óptico es la patología que conduce a la pérdida de visión ${ }^{4}$.

Se estima que aproximadamente 79.6 millones de personas padecieron glaucoma durante 2020 y más de 11 millones tuvieron ceguera bilateral como consecuencia ${ }^{5}$. En Colombia, las consultas por glaucoma se han incrementado en los últimos años, y se reporta una prevalencia del $0.08 \%(35,097)$ en el 2009 y del $0.14 \%$ $(66,229)$ en $2014^{6}$. El departamento del Quindío también ha demostrado un incremento en la prevalencia de glaucoma dentro del periodo 2009-2014, con un 0.08 y un $0.12 \%$, respectivamente, presentando la prevalencia más alta en el año 2013 , de $0.14 \%$.

El presente artículo describe la prevalencia del glaucoma, sus comorbilidades y hallazgos oftalmológicos en pacientes que consultaron a una institución especializada durante el año 2018.

\section{Métodos}

Se planteó un estudio descriptivo de corte transversal.

\section{Población y muestra}

La población fueron todas las historias clínicas de pacientes que consultaron a una institución especializada del Departamento del Quindío, durante el año 2018, con diagnóstico de glaucoma, que tenían la información de las variables del estudio. Se excluyeron historias con datos incompletos.

Del total de pacientes que consultaron en el año $2018(8,164)$, se identificaron los que tenían diagnóstico de glaucoma $(1,036)$ y de estos se tomó una muestra estadísticamente significativa, con un nivel de confianza del $95 \%$ y un margen de error del $5 \%$, para un valor de $\mathrm{n}=227$.

Se tomaron las siguientes variables: edad, sexo, año de primera consulta, controles al año, tiempo entre controles; comorbilidades: hipertensión arterial, diabetes mellitus (DM), dislipidemia; agudeza visual en ambos ojos y presión intraocular (PIO); fondo de ojo: excavaciones del disco óptico; manejo farmacológico y manejo quirúrgico oftálmico.

\section{Procesamiento y análisis de la información}

Se realizó un muestreo aleatorio con reemplazo sistemático en caso de no cumplir con los criterios de inclusión y se tabuló la información obtenida de las historias clínicas en una base de datos diseñada para tal fin por medio del programa Excel ${ }^{\circledR}$.

Se procesó y analizó la información de las variables en el programa StatGraphics Centurión ${ }^{\circledR}$, versión 17.2.04.

Se hizo una prueba de normalidad a las variables y se describieron con medidas de tendencia central. Se hizo un análisis de varianza (ANOVA) para las variables numéricas y una prueba de Chi-cuadrada para las variables categóricas; se consideró una diferencia estadísticamente significativa cuando el valor de $p$ fue $<0.05$.

\section{Consideraciones éticas}

El proyecto fue aprobado por el Comité de Bioética de la Facultad de Ciencias de la Salud, por el Acta $N$ ․o 2 del 8 de febrero de 2019 y se obtuvo el consentimiento informado institucional.

\section{Resultados}

En el año 2018 la prevalencia del glaucoma en la Clínica Oftalmológica del Quindío fue de 1,036 de un total de 8,164 pacientes (12.68\%) que consultaron ese año en este centro de referencia especializado.

Como puede observarse en la tabla 1, la edad promedio fue de 69.97 años y el promedio de controles al año fue de 3.06 .

La PIO promedio fue de $16.55 \mathrm{mmHg}$ en el ojo derecho y de $16.69 \mathrm{mmHg}$ en el ojo izquierdo. La media de las excavaciones del ojo derecho es de 0.60 (intervalo de confianza del 95\% [IC 95\%]: 0.57-0.62) y del ojo izquierdo es de 0.61 (IC 95\%: 0.55-0.60).

Como puede observarse en la tabla 2, el tipo de glaucoma más frecuentemente encontrado fue el glaucoma primario de ángulo abierto (GPAA), con el $66.52 \%$ del total, y se presentó más en mujeres (35.68\%), sin diferencias significativas $(p=0.589)$.

En cuanto a los antecedentes, se encontró que el $57.71 \%$ tiene hipertensión arterial, el $20.26 \%$ de los pacientes tienen DM y el $27.75 \%$ de los pacientes tienen dislipidemia. 
Tabla 1. Variables cuantitativas de los pacientes con glaucoma

\begin{tabular}{|c|c|c|c|}
\hline \multirow[b]{2}{*}{ Variable } & \multicolumn{2}{|c|}{$\begin{array}{l}\text { Glaucoma } \\
(\mathrm{n}=227)\end{array}$} & \multirow[b]{2}{*}{$\begin{array}{l}\text { Valores de } \\
\text { referencia }\end{array}$} \\
\hline & $\begin{array}{c}\text { Promedio } \pm \\
\text { DE }\end{array}$ & IC $95 \%$ & \\
\hline Edad (años) & $69.97 \pm 11.92$ & $68.41-71.53$ & NA \\
\hline $\begin{array}{l}\text { Excavaciones ojo } \\
\text { derecho }\end{array}$ & $0.60 \pm 0.18$ & $0.57-0.62$ & $0.50-0.67$ \\
\hline $\begin{array}{l}\text { Excavaciones ojo } \\
\text { izquierdo }\end{array}$ & $0.61 \pm 0.19$ & $0.59-0.64$ & $0.50-0.67$ \\
\hline $\begin{array}{l}\text { PIO ojo derecho } \\
\text { - PV (mmHg) }\end{array}$ & $16.91 \pm 6.93$ & $15.95-17.88$ & $12-22 \mathrm{mmHg}$ \\
\hline $\begin{array}{l}\text { PIO ojo izquierdo } \\
\text { - PV (mmHg) }\end{array}$ & $17.01 \pm 6.12$ & $16.14-17.87$ & $12-22 \mathrm{mmHg}$ \\
\hline $\begin{array}{l}\text { PIO ojo derecho } \\
\text { - UV (mmHg) }\end{array}$ & $16.55 \pm 5.78$ & $15.72-17.38$ & $12-22 \mathrm{mmHg}$ \\
\hline $\begin{array}{l}\text { PIO ojo izquierdo } \\
\text { - UV (mmHg) }\end{array}$ & $16.69 \pm 5.17$ & $15.95-17.44$ & $12-22 \mathrm{mmHg}$ \\
\hline $\begin{array}{l}\text { Controles al año } \\
\text { (n de controles) }\end{array}$ & $3.06 \pm 2.35$ & $2.75-3.36$ & NA \\
\hline $\begin{array}{l}\text { Tiempo entre } \\
\text { controles (n de } \\
\text { meses) }\end{array}$ & $2.72 \pm 2.26$ & $2.43-3.02$ & NA \\
\hline
\end{tabular}

DE: desviación estándar; IC 95\%: intervalo de confianza del 95\%; PIO: presión intraocular; PV: primera vez; UV: última vez; NA: no aplica.

La agudeza visual más frecuente, reportada en los pacientes con diagnóstico de glaucoma fue 20/30 para el ojo derecho (con un $18.94 \%$ ) y $20 / 40$ para el ojo izquierdo (con una frecuencia del 19.82\%).

En cuanto a las características del manejo del glaucoma, el $84.14 \%$ de los pacientes está recibiendo manejo farmacológico. El medicamento que más se indicó fue el latanoprost, en un $19.82 \%$.

El $40.08 \%$ de los pacientes ha recibido tratamiento quirúrgico (incluyendo iridiotomía), más frecuente en el sexo masculino, con el $20.26 \%$ ( $p=0.000)$.

\section{Discusión}

El presente estudio encontró una prevalencia de glaucoma del $12.68 \%$ durante el año 2018 en un centro especializado de la ciudad de Armenia, Quindío.

Se encontró predominio del sexo femenino en un $54.19 \%$, coincidiendo con lo descrito por Cook, et al., quienes evidenciaron que las mujeres se encuentran más afectadas que los hombres en un $59 \%$ de los casos?.
Tabla 2. Diagnóstico y antecedentes en pacientes con glaucoma

\begin{tabular}{|c|c|c|c|}
\hline Variable & Categoría & $\begin{array}{l}\text { Frecuencia } \\
(\mathrm{n}=227)\end{array}$ & Porcentaje \\
\hline \multirow[t]{2}{*}{ Sexo } & Femenino & 123 & $54.19 \%$ \\
\hline & Masculino & 104 & $45.81 \%$ \\
\hline \multirow[t]{7}{*}{ Diagnósticos } & $\begin{array}{l}\text { Glaucoma } \\
\text { primario de } \\
\text { ángulo abierto }\end{array}$ & 151 & $66.52 \%$ \\
\hline & $\begin{array}{l}\text { Glaucoma } \\
\text { primario de } \\
\text { ángulo cerrado }\end{array}$ & 38 & $16.74 \%$ \\
\hline & $\begin{array}{l}\text { Sospecha de } \\
\text { glaucoma }\end{array}$ & 32 & $14.10 \%$ \\
\hline & $\begin{array}{l}\text { Glaucoma } \\
\text { secundario a } \\
\text { otros trastornos } \\
\text { del ojo }\end{array}$ & 3 & $1.32 \%$ \\
\hline & $\begin{array}{l}\text { Glaucoma no } \\
\text { especificado }\end{array}$ & 1 & $0.44 \%$ \\
\hline & $\begin{array}{l}\text { Glaucoma } \\
\text { secundario a } \\
\text { drogas }\end{array}$ & 1 & $0.44 \%$ \\
\hline & $\begin{array}{l}\text { Glaucoma } \\
\text { secundario a } \\
\text { inflamación } \\
\text { ocular }\end{array}$ & 1 & $0.44 \%$ \\
\hline \multirow{3}{*}{$\begin{array}{l}\text { Antecedente } \\
\text { de } \\
\text { hipertensión } \\
\text { arterial }\end{array}$} & Sí & 131 & $57.71 \%$ \\
\hline & No & 16 & $7.05 \%$ \\
\hline & NR & 80 & $35.24 \%$ \\
\hline \multirow{3}{*}{$\begin{array}{l}\text { Antecedente } \\
\text { de diabetes } \\
\text { mellitus }\end{array}$} & Sí & 46 & $20.26 \%$ \\
\hline & No & 16 & $7.05 \%$ \\
\hline & NR & 165 & $72.69 \%$ \\
\hline \multirow{3}{*}{$\begin{array}{l}\text { Antecedente } \\
\text { de } \\
\text { dislipidemia }\end{array}$} & Sí & 63 & $27.75 \%$ \\
\hline & No & 16 & $7.05 \%$ \\
\hline & NR & 148 & $65.20 \%$ \\
\hline
\end{tabular}

NR: no registra.

El diagnóstico de glaucoma más frecuentemente encontrado en este estudio fue el de GPAA, seguido del diagnóstico de glaucoma primario de ángulo cerrado (GPAC), los cuales se presentaron más en el sexo femenino. Lo cual es similar en cuanto a la prevalencia global, siendo el GPAA el de mayor carga global, con una prevalencia del $3.05 \%$, y el GPAC del $0.50 \%$ en personas entre los $40-80$ años ${ }^{8}$.

Según Cook, et al. ${ }^{7}$, las mujeres están más afectadas que los hombres por GPAA, mientras que Tham, et al. 
encontraron que los hombres presentan un $36 \%$ más de probabilidades de tener GPAA que las mujeres ${ }^{8}$.

En este estudio, la media de la PIO hallada en la última consulta fue de $16.55 \mathrm{mmHg}$ en el ojo derecho y $16.69 \mathrm{mmHg}$ en el ojo izquierdo, es decir, dentro de los rangos normales (que son entre 10 y $21 \mathrm{mmHg}$ ), similar a un estudio japonés denominado Tajimi, que encontró predominio de los valores de la PIO dentro de los rangos normales en la mayoría de los casos, en $21 \mathrm{mmHg}$ o menos en el $92 \%$ de los pacientes con GPAA bajo tratamiento 9 .

Hoy en día, la PIO elevada se define como un factor de riesgo importante para el desarrollo y la progresión del glaucoma y no como un biomarcador de diagnóstico como era hace varias décadas ${ }^{10}$.

Respecto a los antecedentes se encontró en este estudio que el $57.71 \%$ tenía hipertensión arterial. Según Zhao, et al., la hipertensión sistémica puede contribuir al aumento de la PIO por medio de la sobreproducción o la disminución del flujo de humor acuoso, y algunos, pero no todos, los estudios de población han encontrado asociaciones estadísticamente positivas entre la presión arterial sistólica y la presión arterial diastólica con la $\mathrm{PIO}^{11}$.

El segundo antecedente encontrado fue la DM en el $20.26 \%$, siendo predominante en el sexo femenino. En el metaanálisis de estudios de cohorte prospectivos de Zhao, et al. se mostró que la presencia de DM incrementa el riesgo de glaucoma en un 36\% además y evidenció la fuerte asociación existente entre DM y GPAA $^{12}$.

El medicamento más frecuentemente formulado en los pacientes con diagnóstico de glaucoma fue el latanoprost, cumpliendo con las guías internacionales de práctica clínica, las cuales recomiendan como terapia de primera línea las prostaglandinas tópicas ${ }^{4}$. Además, ha demostrado ofrecer un buen efecto hipotensor y es un medicamento que se encuentra incluido dentro del Plan de Beneficios en Salud en Colombia, lo cual lo hace accesible para la población en general, logrando generar mayor adherencia al tratamiento ${ }^{13}$.

Según una caracterización epidemiológica del glaucoma en una institución oftalmológica de Bogotá realizada por la Universidad Nacional, se encontró similitud en los resultados donde el medicamento mayormente formulado fue el latanoprost, además del timolol ${ }^{13}$.

Se observó un aumento en el número de consultas entre los años 2016 y 2018. Según el Análisis de Situación de Salud Visual (ASIS) en Colombia del año 2016, las consultas con diagnóstico de glaucoma han ido en ascenso desde el año 2009 con una prevalencia del $0.08 \%$, al 2014 con una prevalencia del $0.14 \%$.

Dicho aumento en el diagnóstico podría haberse dado gracias al uso de la tomografía óptica de coherencia, ya que ha surgido a lo largo de los años con la capacidad de detectar cambios en la cabeza del nervio óptico, la capa de fibra nerviosa retiniana y de células ganglionares, mucho antes de que los defectos se manifiesten funcionalmente ${ }^{14}$.

Esta situación podría explicar el aumento paulatino de este diagnóstico en este centro, como un logro importante del desarrollo e implementación de las RIAS en el territorio colombiano.

Sin embargo, se necesitan más estudios epidemiológicos prospectivos que permitan establecer las causales del incremento de casos de enfermedades de los ojos, así como el impacto de las rutas y las guías de atención en la gestión de dicho riesgo.

\section{Agradecimientos}

A la Clínica oftalmológica Quindío, a la Dra. Stella Hincapié por facilitar todas las condiciones para la recolección de la información, y a la Dra. Diana Marcela Curtidor por su orientación en los aspectos bioéticos.

\section{Financiamiento}

La presente investigación no ha recibido ninguna financiación o beca específica de agencias de los sectores público, comercial, o con ánimo de lucro.

\section{Conflicto de intereses}

Los autores declaran no tener conflicto de intereses.

\section{Responsabilidades éticas}

Protección de personas y animales. Los autores declaran que para esta investigación no se han realizado experimentos en seres humanos ni en animales.

Confidencialidad de los datos. Los autores declaran que han seguido los protocolos de su centro de trabajo sobre la publicación de datos de pacientes.

\section{Derecho a la privacidad y consentimiento infor-} mado. Los autores han obtenido el consentimiento informado de los pacientes $y / 0$ sujetos referidos en el artículo. Este documento obra en poder del autor de correspondencia. 


\section{Bibliografía}

1. Rutas integrales de atención en Salud (RIAS). Rutas Integrales de Atención en Salud reglamentadas por la Resolución 3202 del año 2016 [Internet]. Colombia: Ministerio de Salud y Protección Social; 2016. Disponible en: https:// www.minsalud.gov.co/Paginas/rutas- integrales-de-atencion-en-salud.aspx

2. Guía de Práctica Clínica para el manejo de la hipertensión arterial primaria (HTA) [Internet]. Colombia: Ministerio de Salud y Protección Social; 2017. Disponible en: https://scc.org.co/wp-content/uploads/2018/02/ GPC HTA-MINSALUD-2017.pdf

3. Guía de práctica clínica para el diagnóstico, tratamiento y seguimiento de la diabetes mellitus tipo 2 en la población mayor de 18 años [Internet]. Colombia: Ministerio de Salud y Protección Social; 2015. Disponible en: http://repositorio.mederi.com.co/handle/123456789/363

4. Guías del Consejo Internacional de Oftalmología (ICO) para el Glaucoma [Internet]. International Council of Ophtalmology; 2015. Disponible en http://www.icoph.org/downloads/ICOGlaucomaGuidelines-Spanish.pdf

5. Gupta D, Chen PP. Glaucoma. Am Fam Physician. 2016;93(8):668-74.

6. Análisis de Situación de Salud Visual en Colombia 2016. Conv 519 del 2015 [Internet]. Colombia: Ministerio de Salud y Protección Social; 2016. Disponible en: https://www.minsalud.gov.co/sites/rid/Lists/BibliotecaDigital/RIDE/VS/PP/ENT/asis-salud-visual-colombia-2016.pdf

7. Cook C, Foster P. Epidemiology of glaucoma: What's new? Can J Ophthalmol. 2012;47(3):223-26.
8. Tham YC, Li X, Wong TY, Quigley HA, Aung T, Cheng CY. Global prevalence of glaucoma and projections of glaucoma burden through 2040: A systematic review and meta-analysis. Ophthalmology. 2014;121(11):2081-90

9. Suzuki Y, Iwase A, Araie M, Yamamoto T, Abe H, Shirato S, et al. Risk factors for open-angle glaucoma in a Japanese population. The Tajimi Study. Ophthalmology. 2006;113(9):1613-17.

10. Leeman M, Kestelyn P. Glaucoma and blood pressure. Hypertension. 2019;73(5):944-50.

11. Zhao D, Cho J, Kim MH, Guallar E. The association of blood pressure and primary open-angle glaucoma: A meta-analysis. Am J Ophthalmol. 2014;158(3):615-27.e9.

12. Zhao YX, Chen XW. Diabetes and risk of glaucoma: Systematic review and a meta-analysis of prospective cohort studies. Int $\mathrm{J}$ Ophthalmol. 2017;10(9):1430-35.

13. Pineda Gutiérrez LM. Caracterización epidemiológica del glaucoma en una institución oftalmológica de Bogotá [tesis en Internet]. [Bogotá]: Universidad Nacional de Colombia, Facultad de Medicina, Unidad de Oftalmología; 2017. Disponible en: https://repositorio.unal.edu.co/bitstream/handle/unal/62873/1088268401.2017. pdf? sequence $=1 \&$ is Allowed=y

14. Sahoo B. Role of optical coherence tomography in the evaluation and management of glaucoma. En: Lanza JPE-M, editor. Rijeka: IntechOpen; 2019. 